\title{
Review of the Existing Game Based Learning System in Cybersecurity
}

\author{
Tolulope Awojana \\ Department of Technology Systems \\ College of Engineering and Technology \\ East Carolina University \\ Greenville, NC 27858 \\ awojanat17@students.ecu.edu
}

\author{
Te-Shun Chou \\ Department of Technology Systems \\ College of Engineering and Technology \\ East Carolina University \\ Greenville, NC 27858 \\ chout@students.ecu.edu
}

\author{
Hempenius Nicholas \\ Department of Technology Systems \\ College of Engineering and Technology \\ East Carolina University \\ Greenville, NC 27858 \\ hempeniusn15@students.ecu.edu
}

\begin{abstract}
As the world is advancing in technology, there is an increasing trend in the number of malware attacks recorded. Estimated losses from cyber-crime and cyber espionage amount to hundreds of billions annually. The need to build, maintain, and defend computing resources is greater than ever before. Gamebased learning are competitive, interactive lessons which allows the learner to have fun while gaining knowledge by using specific skills to achieve definite outcomes.
\end{abstract}

\section{Keywords}

Cybersecurity; Games; Players; Attacks; Defense; Pedagogy;

\section{INTRODUCTION}

Game based learning is commonly used as a method of learning in the society today. Eames defined this method of learning as the incorporation of games which could imply video games in instructions [1]. Games are often times played for the sole purpose of fun, for taking up challenges and to outplay the other opponents. With the evolvement of the various disciplines over time, there is a constant shift in the view of threats and vulnerabilities in cybersecurity today. Hence, it has become important to incorporate a game based learning system into the field of cybersecurity so as to engage the mind of the young adults thereby establishing a foundation of the skills required in information security and creating a form of passion within them to train them to become the next generation of cybersecurity professionals while having fun alongside. This presentation reviews the different applications used for game based learning system in cybersecurity and possible ways to improve on the existing systems.

\section{LITERATURE REVIEW}

Several studies on the game based learning system in Cybersecurity have highlighted its effectiveness and shown how skills and competencies are being improved through this learning process. The existing systems were classified into 3 different categories: Category 1 involves only the theoretical description of themes and concepts of cybersecurity without a representation of the actual gameplay. Game based systems in this category includes: Control-Alt-Hack, Stop That Post Game

Permission to make digital or hard copies of part or all of this work for personal or classroom use is granted without fee provided that copies are not made or distributed for profit or commercial advantage and that copies bear this notice and the full citation on the first page. Copyrights for third-party components of this work must be honored. For all other uses, contact the Owner/Author.

SIGITE '18, October 3-6, 2018, Fort Lauderdale, FL, USA

(c) 2018 Copyright is held by the owner/author(s).

ACM ISBN 978-1-4503-5954-2/18/10.

https://doi.org/10.1145/3241815.3241839
[2] and Shadowrun Returns. Category 2 (One Player Game) involves the games played by one person where the individual would be tested based on the understanding of cybersecurity concepts introduced in the application. Game based systems in this category includes: CyberProtect, CyberAware and CyberCIEGE.[2]. Category 3 (Multi Player Game) involves competition between the different individuals or teams with reward based outcomes based on understanding of cybersecurity concepts. Game based systems in this category includes: CyberNEXS, InCTF (India Capture The Flag) and GenCyber.[2][3][4]

\section{CONCLUSION}

Based on the classifications of the existing games highlighted above, awareness, defensive and attacker strategies were drawn out as features to identify the contributions of the model to the field of cybersecurity. Valid tools have been developed to consolidate and enhance learning and the authors of each tools assessed their tools through the awareness, defensive and attacker strategies. The multiplayer game approach can be identified as one of the best interactive medium for game based learning in the field of cybersecurity. However, a collaboration of the above mentioned systems with better improvement on the scalability, flexibility and increased use of themes can equip and prepare students on cybersecurity education. An improvement on the existing game based learning system is recommended to include the added features on awareness, defensive and attacker strategies.

\section{ACKNOWLEDGMENTS}

This research is made possible by the National Science Foundation under grant 1723650. The authors are grateful to the support of Department of Technology Systems in the College of Engineering and Technology at East Carolina University.

\section{REFERENCES}

[1] Eames, J. (2014). What Game-Based Learning Can Do for Student Achievement. EdSurge.

[2] Gestwicki, P., \& Stumbaugh, K. (2015). Observations and Opportunities in Cybersecurity Education Game Design. IEEE Conference on Computer Games: AI, Animation, Mobile, Multimedia, Educational and Serious Games. DOI:10.1109/CGames.2015.7272970.

[3] Boopathi, K., Sreejith, S. \& Bithin, A. (2015). Learning Cyber Security Through Gamification. Indian fournal of Science and Technology, Vol. 8(7), 642-649.

[4] Raman, R., Lal, A. \& Achuthan, K. (2014). Serious Games Based Approach to Cyber Security Concept Learning: Indian Context. Semantic Scholar. 